\title{
Cognitive decline associated with dementia and type 2 diabetes: the interplay of risk factors
}

\author{
C. Messier • M. Gagnon
}

Received: 21 August 2009 /Accepted: 26 August 2009/Published online: 25 September 2009

(C) Springer-Verlag 2009

Keywords Ageing Alzheimer's disease $\cdot A P O E$ \&4 . Cognition · Dementia $\cdot$ Lipoprotein · Memory

Vascular dementia

The possible association between type 2 diabetes and dementia, Alzheimer's disease in particular, has been shrouded in confusion for some time, with some researchers noting that Alzheimer's patients are rarely diabetic [1] but others suggesting diabetes to be a major risk factor for the disease [2]. Since an earlier review of the evidence [3], a number of things have become clearer. First, histopathological examinations of the brains of type 1 and type 2 diabetic patients have demonstrated that diabetes as such does not produce the amyloid plaques and neurofibrillary tangles considered hallmarks of Alzheimer's disease [4]-a finding supported by a recent extensive post-mortem examination [5]. Second, research has shown that, in nondemented patients with type 2 diabetes, cognitive deficits are relatively mild before the age of 70 years, although they increase in frequency and severity thereafter [6]. Finally, people with type 2 diabetes who maintain less than optimal glycaemic control (i.e. with many occurrences of high blood glucose levels) are more likely to start manifesting cognitive deficits. Interestingly, improving glycaemic control in this

\section{Messier $(\square)$}

School of Psychology, University of Ottawa,

200 Lees Avenue, Room E260J,

Ottawa, ON, Canada K1N 6N5

e-mail: cmessier@uottawa.ca

\section{Gagnon}

Riverside Professional Medical Center,

1919 Riverside Drive, Room 306,

Ottawa, ON, Canada K1H 1A2 population can reduce these cognitive deficits [7], suggesting that cognitive deficits might not be permanent in patients in whom presentation is early.

Unfortunately, for a considerable number of patients, the presence of diabetes increases the impact of other risk factors that also lead to cognitive decline and dementia (see Fig. 1). For example, hypertension [8] and arterial disease [9], which are found in many older people with type 2 diabetes, can contribute alone or in concert to decreased cognitive performance and, eventually, dementia. Another risk factor for cognitive decline in ageing and dementia concerns the gene encoding apolipoprotein $\mathrm{E}(A P O E)$. This gene has three major alleles ( $A P O E \varepsilon 2, \varepsilon 3$ and $\varepsilon 4)$, which produce three distinct variants of apolipoprotein E. APOE is a glycoprotein attached to lipoprotein particles that acts as a ligand for the receptor-mediated endocytosis (transport) of lipoproteins particles in the brain from which cholesterol is released in the brain. People with two $A P O E$ \&2 alleles have a higher risk of premature vascular disease but a lower risk of Alzheimer's disease. However, people with one $A P O E$ \& 4 allele have a two- to threefold higher risk of developing Alzheimer's disease, while people with two $A P O E$ \&4 alleles have a 12-fold higher risk [10]. APOE \&4 alleles are also associated with early cognitive decline and early manifestation of Alzheimer's disease pathology [11].

Contrary to some previous reports, the largest histological post-mortem study to date found that type 2 diabetes does not increase amyloid deposition or neurofibrillary tangles in diabetic patients with $A P O E$ \&4 alleles [5]. Thus, the increased likelihood of cognitive deficits in diabetic patients with $A P O E$ \&4 alleles is not due to diabetes increasing Alzheimer's disease pathology. Rather, it is more likely that the cerebrovascular lesions associated with diabetes combine synergistically with pathologies associated with Alzheimer's disease to hasten cognitive decline [9]. 


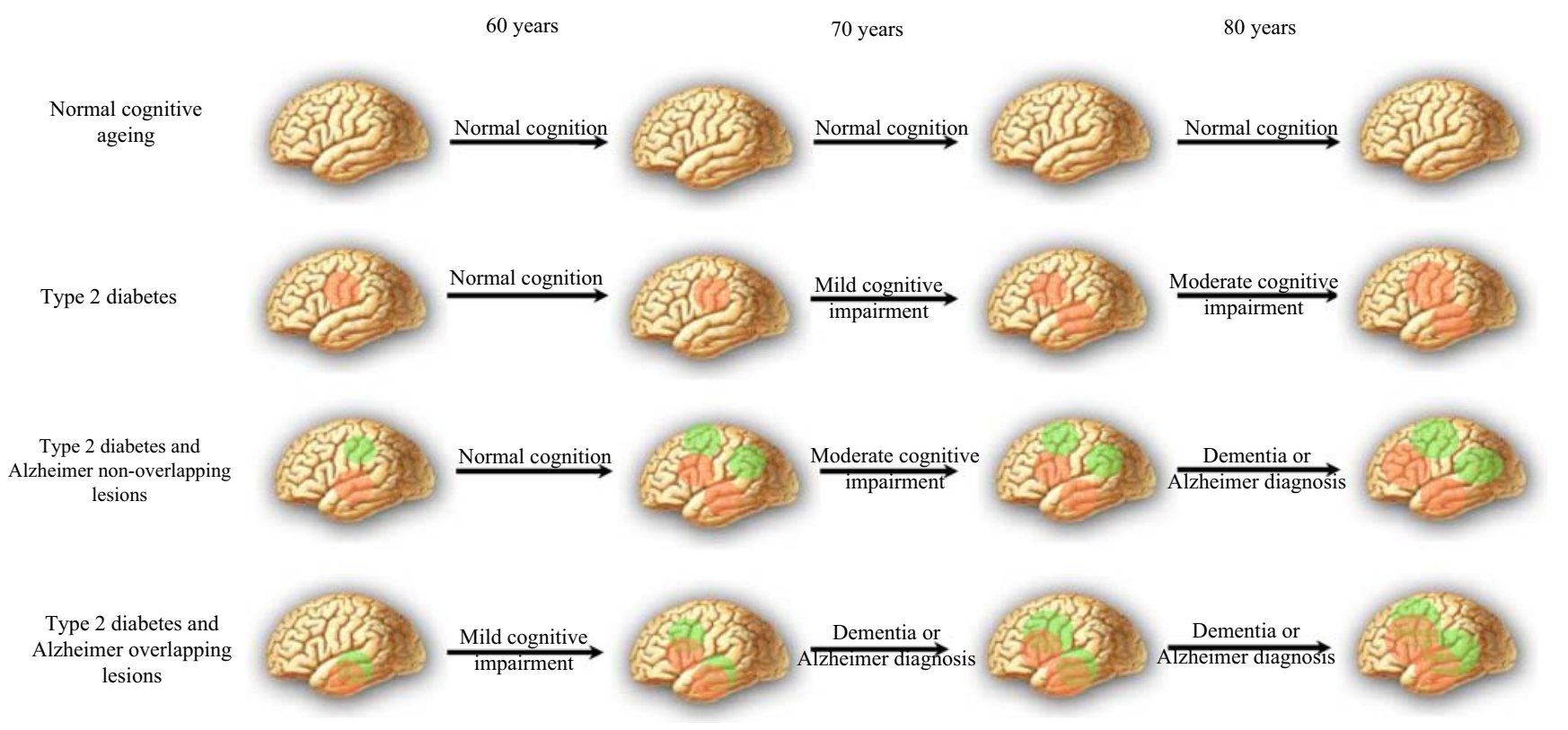

Fig. 1 Progression of cognitive decline as a function of the presence of diabetes and Alzheimer's disease lesions. Diabetes leads to mild cognitive deficits around the seventh decade, probably associated with vascular pathology. Non-overlapping brain lesions due to type 2 diabetes and Alzheimer's disease produce earlier and more pronounced cognitive deficits that progress to dementia. Overlapping lesions produced by type 2 diabetes and Alzheimer's disease produce the greatest and most extensive cognitive deficits and lead to the fastest progression towards dementia. The pink shading indicates vascular lesions and the green shading indicates amyloid and neurofibrillary tangles
Taken together, these observations suggest that while type 2 diabetes as such may not cause large decrements in cognitive function, it may increase the impairing effects of other risk factors, such as hypertension, arterial disease and $A P O E \varepsilon 4$ genotype. Knowing how these different risk factors overlap and how they interplay would be of great help in predicting the course of cognitive changes in people with multiple risk factors for dementia. Moreover, a diabetic person who has one or two $A P O E \& 4$ alleles would be even more strongly encouraged to engage in the difficult lifestyle changes known to modulate the course of diabetes and vascular disease.

The study by Dore and colleagues [12] presented in this issue of Diabetologia was designed to address the question of how the risks of different factors add up to predict early cognitive deficits in type 2 diabetes. Few studies have combined APOE genotyping with a measure of diabetes and extensive cognitive testing to evaluate this issue. Dore's large cross-sectional community-based study observed more cognitive deficits in people with diabetes and in carriers of the $A P O E \& 4$ allele. Interestingly, when present in combination, diabetes and the $A P O E \varepsilon 4$ genotype acted synergistically to produce a cognitive decline that was beyond a simple additive effect of each risk factor alone. The large potentiation produced by the combination of these two risk factors for cognitive decline is likely due to the older age of their cohort and the inclusion of people with mild cognitive impairment. The major limitation of the study by Dore et al. is the cross-sectional nature of the design, which reduces its ability to predict the progression of cognitive decline associated with the presence of risk factors. In addition, the low number of African-American participants may have precluded the observation that, in this subset of the population, type 2 diabetes does not appear to increase the cognitive decline associated with the APOE \&4 genotype, as previously reported in a study of a younger cohort (mean age 55 years) [13]. However, a follow-up study of the same cohort did not report such lack of association [14], leaving the question unanswered as to whether $A P O E \& 4$ genotype in African-Americans has the same consequences for cognitive decline.

The cause of the accelerated cognitive decline in type 2 diabetic patients is still subject to debate. Early hypotheses (i.e. advanced glycation end-products) suggested that brain cells were exposed to high glucose levels. This is unlikely because brain glucose levels are $20-30 \%$ of blood levels [15]. An asymmetry in glucose transporters on each side of the blood-brain barrier prevents glucose levels in the brain from reaching those observed in blood that are associated with diabetes pathology in the rest of the body. However, while the brain side of the brain blood vessels is protected from the high glucose levels observed in diabetes, the inner walls of the brain vasculature (on the blood side) are exposed to hyperglycaemia, which can cause vascular damage. Other hypotheses propose that putative changes 
in brain insulin levels or sensitivity in the diabetic patients facilitate amyloid deposition and neurofibrillary tangles, but these ideas also appear unlikely at this time [16]. A more likely hypothesis to explain accelerated cognitive decline is the higher prevalence of vascular disease in diabetes, some of which may be caused by high blood glucose levels or by other associated pathologies, such as hypertension and atherosclerosis.

Contrary to the clearer association between diabetes and vascular dementia, the question of whether diabetes increases the likelihood of developing Alzheimer's disease has yet to be answered, with some studies showing that having both diabetes and $A P O E \& 4$ genotype increases the risk of a diagnosis of probable Alzheimer's disease or mixed dementia [17, 18], while others do not [19, 20]. This lack of consensus is driven on the one hand by the difficulty in interpreting and classifying the observations of vascular damage using imaging techniques or postmortem examinations [21], and on the other hand by the relative lack of correlation between mild diffuse vascular damage and cognitive deficits. One of the possible ways diabetes might increase the likelihood of a diagnosis of Alzheimer's disease is by creating small-vessel-associated lesions [22]. If, in a given patient, these lesions occur in the brain regions that are most affected by Alzheimer's disease, then larger deficits in the cognitive functions controlled by these regions would be observed. This occurrence would eventually lead to an earlier diagnosis of Alzheimer's disease. A second source of variability might arise from the observation that few deficits are observed before the age of 70 in non-demented diabetic patients [6]. Variation in the mean age of participants in studies might also lead to different conclusions. Finally, not all patients can achieve good glycaemic control, and some studies suggest that worse glycaemic control is associated with worse cognition [9]. Thus, variability in participants' glycaemic control across studies may also lead to different conclusions. Studies that incorporate new in vivo imaging techniques of amyloid deposits and the evaluation of infarcts together with the measure of regional brain volume will likely provide a more definitive picture of the interaction between type 2 diabetes and dementia.

To summarise, type 2 diabetes can lead to cognitive deficits later in life (earlier if there is poor glycaemic control). The most likely cause of cognitive deficits in later life in diabetic patients is vascular disease; if vascular disease damage overlaps with the damage produced by other diseases such as Alzheimer's disease, the cognitive effects will be cumulative and this may hasten the progression of Alzheimer's disease or other dementia. On the positive side, there are indications that exercise, weight control, and glycaemic control can afford protection against cognitive deficits as they do against other diabetic compli- cations. This knowledge may help to motivate patients to make greater efforts to follow these prescriptions.

Duality of interest The authors declare that there is no duality of interest associated with this manuscript.

\section{References}

1. Nielson KA, Nolan JH, Berchtold NC, Sandman CA, Mulnard RA, Cotman CW (1996) Apolipoprotein-E genotyping of diabetic dementia patients: is diabetes rare in Alzheimer's disease? [see comments]. J Am Geriatr Soc 44:897-904

2. Messier C (2003) Diabetes, Alzheimer's disease and apolipoprotein genotype. Exp Gerontol 38:941-946

3. Messier C, Gagnon M (1996) Glucose regulation and cognitive functions: relation to Alzheimer's disease and diabetes. Behav Brain Res 75:1-11

4. Heitner J, Dickson D (1997) Diabetics do not have increased Alzheimer-type pathology compared with age-matched control subjects. A retrospective postmortem immunocytochemical and histofluorescent study. Neurology 49:1306-1311

5. Alafuzoff I, Aho L, Helisalmi S, Mannermaa A, Soininen H (2009) beta-Amyloid deposition in brains of subjects with diabetes. Neuropathol Appl Neurobiol 35:60-68

6. Ryan CM, Geckle M (2000) Why is learning and memory dysfunction in type 2 diabetes limited to older adults? Diabetes Metab Res Rev 16:308-315

7. Ryan CM, Freed MI, Rood JA, Cobitz AR, Waterhouse BR, Strachan MW (2006) Improving metabolic control leads to better working memory in adults with type 2 diabetes. Diabetes Care 29:345-351

8. van den Berg E, Kloppenborg RP, Kessels RP, Kappelle LJ, Biessels GJ (2009) Type 2 diabetes mellitus, hypertension, dyslipidemia and obesity: a systematic comparison of their impact on cognition. Biochim Biophys Acta 1792:470-481

9. Tiehuis AM, Mali WP, van Raamt AF et al (2009) Cognitive dysfunction and its clinical and radiological determinants in patients with symptomatic arterial disease and diabetes. J Neurol Sci 283:170-174

10. Kim J, Basak JM, Holtzman DM (2009) The role of apolipoprotein E in Alzheimer's disease. Neuron 63:287-303

11. Craft S, Teri L, Edland SD et al (1998) Accelerated decline in apolipoprotein E-epsilon4 homozygotes with Alzheimer's disease. Neurology 51:149-153

12. Dore G, Elias M, Robbins M, Elias P, Nagy Z (2009) Presence of the APOE $\varepsilon 4$ allele modifies the relationship between type 2 diabetes and cognitive performance: the Maine-Syracuse Study. Diabetologia. doi:10.1007/s00125-00009-01497-00122

13. Blair CK, Folsom AR, Knopman DS, Bray MS, Mosley TH, Boerwinkle E (2005) APOE genotype and cognitive decline in a middle-aged cohort. Neurology 64:268-276

14. Knopman DS, Mosley TH, Catellier DJ, Coker LH (2009) Fourteenyear longitudinal study of vascular risk factors, APOE genotype, and cognition: the ARIC MRI Study. Alzheimers Dement 5:207-214

15. Abi-Saab WM, Maggs DG, Jones T et al (2002) Striking differences in glucose and lactate levels between brain extracellular fluid and plasma in conscious human subjects: effects of hyperglycemia and hypoglycemia. J Cereb Blood Flow Metab 22:271-279

16. Craft S, Watson GS (2004) Insulin and neurodegenerative disease: shared and specific mechanisms. Lancet Neurology 3:169-178

17. Irie F, Fitzpatrick AL, Lopez OL et al (2008) Enhanced risk for Alzheimer disease in persons with type 2 diabetes and APOE epsilon4: the Cardiovascular Health Study Cognition Study. Arch Neurol 65:89-93 
18. Peila R, Rodriguez BL, Launer LJ (2002) Type 2 diabetes, APOE gene, and the risk for dementia and related pathologies-The Honolulu-Asia Aging Study. Diabetes 51:1256-1262

19. Akomolafe A, Beiser A, Meigs JB et al (2006) Diabetes mellitus and risk of developing Alzheimer disease: results from the Framingham Study. Arch Neurol 63:1551-1555

20. MacKnight C, Rockwood K, Awalt E, McDowell I (2002) Diabetes mellitus and the risk of dementia, Alzheimer's disease and vascular cognitive impairment in the Canadian Study of Health and Aging. Dement Geriatr Cogn Disord 14:77-83

21. Jellinger KA (2008) The pathology of "vascular dementia": a critical update. J Alzheimers Dis 14:107-123

22. Thompson CS, Hakim AM (2009) Living beyond our physiological means: small vessel disease of the brain is an expression of a systemic failure in arteriolar function: a unifying hypothesis. Stroke 40:e322-e330 